\title{
Targeted Transgenic RNAi Knockdown of Cell Fate Determinants Induces Neoplastic Tumor Growth and Metastasis in a Drosophila Transplantation Model of Neural Stem Cell Derived Cancer
}

\author{
Anne-Sophie Laurenson*, Nidhi Saini, Yanrui Jiang and Heinrich Reichert
}

Biozentrum, University of Basel, Klingelbergstrasse 50, Basel, Switzerland

\begin{abstract}
Genetic models such as Drosophila have sophisticated transgenic and molecular genetic tools available to investigate proliferation control in normal and tumorigenic neural stem cells. In this report, we adapted a targeted transgenic RNAi knockdown approach based on the Gal4/UAS expression system to the study of neoplastic tumor formation and metastatic growth in the Drosophila brain tissue transplantation model. Transgenic RNAi driven knockdown of numb, brain tumor (brat) and prospero (pros) in all neuroblasts (type I and type II) resulted in a high incidence of neoplastic tumor formation after transplantation that was comparable to that of loss-of-function mutations in these cell fate determinants. RNAi knockdown of numb and brat specifically restricted to type II neuroblast lineages also resulted in tumor formation after transplantation. A marked temperature dependence of tumor formation after transplantation was documented and quantified for RNAi-induced knockdown of numb, brat and pros. An in vivo assay for micrometastasis formation in ovarioles revealed significant metastatic potential of transplanted overproliferating brain tissue induced by RNAi knockdown of these cell fate determinants. These findings establish the foundation for RNAi-based investigations of the mechanisms which underlie the proliferation, invasion and metastastic potential of neural stem cell induced tumors in the Drosophila model.
\end{abstract}

Keywords: Neural Stem Cells; Neuroblasts; Drosophila; RNAi; Brain Tumors; Brat; Numb; Prospero

Abbreviations: INPs: Intermediate Neural Precursors; GMC: Ganglion Mother Cell; Brat: Brain tumor; Pros: Prospero; wor-Gal4, ase-Gal80: worniu-Gal4, asense-Gal80; insc-Gal4: inscuteable-Gal4

\section{Introduction}

A central feature of stem cells is their ability to generate copies of themselves while giving rise to more differentiated progeny. In consequence, stem cells must avoid cell cycle exit and differentiation while simultaneously avoiding uncontrolled proliferation and tumor formation. As errors in stem cell division rate or in the fine balance between self-renewal and differentiation can result in tumorigenesis, stem cells might be the cells of origin of certain human cancers ("tumor stem cells") [1-3]. The possible contribution of dysregulated stem cells to tumor formation is especially relevant for neural stem cells and the development of neural stem-cell based regenerative therapies for neurological diseases. Self-renewal and amplification of progeny number are known to be striking features of many neural stem cells in the mammalian brain [4-6]. Clearly, both features make neural stem cells promising for transplant-based regenerative therapy, however, they are also potentially hazardous and prone to cancerous dysregulation having devastating results in potential recipient patients [7]. Genetic model systems have been useful in determining the possible contribution of normal and abnormal stem cells to the initiation of cancer and in identifying the molecular events that might drive such transformation [8-11]. Moreover, in genetic model systems such as Drosophila, sophisticated transgenic and molecular genetic tools are now available to investigate the fundamental problem of proliferation control in normal and tumorigenic neural stem cells. Recent results obtained on Drosophila neural stem cells, also called neuroblasts, imply a causative link between impaired neural stem-cell division and brain tumor formation in this genetic model $[10,12,13]$.

Drosophila neuroblasts can be broadly categorized as type I and type II, both of which have a distinct mode of proliferation. Type I neuroblasts proliferate through self-renewing divisions that also give rise to a smaller daughter cell called a Ganglion Mother Cell (GMC) which further only divides once to generate two neural progeny. Type II neuroblast lineages on the other hand proliferate through selfrenewing divisions that give rise to intermediate neural precursors (INPs), each of which undergo limited rounds of self-renewing division that also generate a GMC. Recent work on both types of neuroblasts has provided an insight into the asymmetric cell division process, whereby the unequal distribution of cell fate determinants leads to the generation of daughter cells with different fates (for recent reviews see $[9,11,14-16])$. Three important asymmetrically segregated cell fate determinants have been analysed in greater detail. The first is Numb, a tissue-specific repressor of the Notch pathway [17]. The second is Prospero (Pros), a homeodomain transcription factor that can act as a transcriptional activator and repressor $[17,18]$. The third is Brain tumor (Brat), a member of the NHL domain family involved in translational regulation and cell growth inhibition [19-21]. Consistent with the functions of these genes in repressing growth and self-renewal, loss of either pros, brat or numb results in neuroblast lineages that escape differentiation. This causes overgrowth characterized by the

*Corresponding author: Anne-Sophie Laurenson, Biozentrum, University of Basel, Klingelbergstrasse 50, Basel, Switzerland, Tel: +4161 26716 12; E-mail: anne-sophie.laurenson@unibas.ch

Received December 15, 2011; Accepted January 09, 2011; Published January 11, 2012

Citation: Laurenson AS, Saini N, Jiang Y, Reichert H (2012) Targeted Transgenic RNAi Knockdown of Cell Fate Determinants Induces Neoplastic Tumor Growth and Metastasis in a Drosophila Transplantation Model of Neural Stem Cell Derived Cancer. J Stem Cell Res Ther S12:002. doi:10.4172/2157-7633.S12-002

Copyright: () 2012 Laurenson AS, et al. This is an open-access article distributed under the terms of the Creative Commons Attribution License, which permits unrestricted use, distribution, and reproduction in any medium, provided the original author and source are credited. 
Citation: Laurenson AS, Saini N, Jiang Y, Reichert H (2012) Targeted Transgenic RNAi Knockdown of Cell Fate Determinants Induces Neoplastic Tumor Growth and Metastasis in a Drosophila Transplantation Model of Neural Stem Cell Derived Cancer. J Stem Cell Res Ther S12:002. doi:10.4172/2157-7633.S12-002

Page 2 of 8

unregulated overproduction of neuroblast-like cells at the expense of differentiated neurons [19-22].

Importantly, transplantation of the corresponding mutant brain tissue into wild type hosts results in immortalized transformed cells as well as lethal malignant neoplastic overgrowth, and the resulting tumors can even be successively re-implanted into new hosts for many years [23]. Thus for these three determinants, the connection between self-renewing asymmetric stem cell division and tumorigenicity supports the hypothesis that impaired cell-fate determination is a major cause of cancerous overgrowth in neural stem cell lineages [24,25]. A current challenge is to understand the molecular mechanisms that link aberrant asymmetric neural stem cell divisions to the type of uncontrolled, immortalized cell proliferation that characterize these transplantation-induced tumors in the Drosophila model. This molecular understanding will be crucial for designing and testing novel strategies aimed at preventing neural stem cell-derived brain tumors, which can subsequently be assessed in comparative molecular genetic studies performed on mammalian neural stem cells in vitro and in vivo before translation to human stem cell transplantation therapy.

The recent establishment of transgenic RNA interference (RNAi) technology together with the availability of genome-wide UAS-RNAi libraries in Drosophila makes it possible to extensively and rapidly investigate gene function in complex developmental and pathological processes in a cell- as well as tissue-specific manner in intact organisms [26-28]. Moreover, since RNAi expression is under Gal4/UAS control, it can be targeted in a tissue-specific manner by any of the numerous available Gal4 strains, including an inscuteable-Gal4 (insc-Gal4) line which drives expression in all of the brain neuroblasts and a worniuGal4 driver combined with an asense-Gal80 repressor (wor-Gal4, ase-Gal80) which drives expression specifically in type II neuroblast lineages $[20,29,30]$. The power of this type of transgenic RNAi approach for understanding neuroblast self-renewal and proliferation has recently been demonstrated in a landmark investigation of the network of functionally interacting genes that control cytokinesis, cell growth and differentiation in the Drosophila brain [30].

In this report, we apply the targeted transgenic RNAi knockdown approach used to investigate neuroblast overproliferation in the developing brain to the study of neoplastic tumor formation and metastatic growth in the Drosophila brain tissue transplantation model. We first confirm that RNAi knockdown of numb, brat and pros in all brain neuroblasts (using the insc-Gal4 driver) or specifically in type II brain neuroblast lineages (using the wor-Gal4, ase-Gal80 drivers) results in overproliferation in the intact larval brain. We then transplant the corresponding RNAi knockdown brain tissue into wild type hosts and assay for tumor formation. Our findings show that overproliferating brain tissue containing insc-Gal4 driven RNAi knockdown of numb, brat or pros in all neuroblasts results in a remarkably high incidence of neoplastic tumor formation after transplantation, and we quantify the frequency of these tumor formations for each of the three cell fate determinants. We then show that overproliferating brain tissue containing wor-Gal4, ase-Gal80 driven RNAi knockdown of numb or brat specifically in type II neuroblast lineages also results in tumor formation after transplantation. A marked temperature dependence of tumor formation after transplantation of overproliferating brain tissue is documented and quantified for RNAi-induced knockdown of numb, brat and pros. Subsequently we use an in vivo assay for micrometastasis formation in ovarioles to investigate the metastatic potential of transplanted overproliferating brain tissue induced by RNAi knockdown of numb, brat and pros. These studies form the basis for future RNAi-based investigations of the molecular mechanisms which underlie the immortalized proliferation, invasive behavior and metastatic potential of neural stem cell induced tumors as well as for exploring molecular genetic manipulations which might prevent tumorigenesis in the Drosophila model.

\section{Materials and Methods}

\section{Fly stocks}

All Drosophila stocks were maintained on standard yeast-cornmealagar medium at optimum temperature of $25^{\circ} \mathrm{C}$ and on a $12: 12 \mathrm{~h}$ light/ dark cycle. Fly stocks carrying an inducible UAS-RNAi construct and both the driver lines UAS-Dicer-2; insc-Gal4, UAS-CD8::GFP or UASDicer-2; wor-Gal4, ase-Gal80; UAS-CD8::GFP were obtained from the Vienna Drosophila RNAi Center (VDRC) [30]. brat ${ }^{\mathrm{k} 06028}$ mutants were obtained from Bloomington Stock Center. $w^{1118}$ flies were used as host flies as they provide a good contrast and enable easy visualization of GFP labeled transplanted brain tissue and subsequent tumor formation.

\section{Dissection and visualization of larval brains}

Third instar larval brains were dissected in ice-cold Phosphate Buffer Saline (PBS, Sigma 081M8207) and fixed in $2 \%$ paraformaldehyde (Riedel-de Haen, Sigma-Aldrich, CAS nr. 30525-89-4) for $1 \mathrm{~h}$ at room temperature, washed several times in PBS/0.5\% Triton X-100 (Sigma), and then several times in PBS before being incubated in Vectashield (Vector Laboratories, Inc. Reactolab S.A., H-1000) overnight at $-20^{\circ} \mathrm{C}$. Then larval brains were neatly dissected and were mounted onto a slide in Vectashield mounting medium. Pictures of GFP-positive larval brains (direct-GFP), both in control and overproliferating larval brains were taken using a Leica TCS SP5 confocal microscope and the images were processed using standard Fiji [31] or ImageJ 1.42a (NIH, USA).

\section{Transplantation of larval brain pieces}

Males carrying a Gal-4 inducible UAS-RNAi construct were crossed to virgin flies of the respective driver lines. Crosses were set up at $29^{\circ} \mathrm{C}$ and third-instar larvae were collected after 5-6 days and transplantations of GFP-positive, larval brain pieces were performed as previously described [23,32], with minor modifications. Freshly enclosed host flies were collected and allowed to age at $25^{\circ} \mathrm{C}$ such that they were 3-4 days old at the time of transplantation. These $w^{1118}$ female adult hosts were anesthetized by $\mathrm{CO}_{2}$ and immobilized on a metal plate kept on ice, with double-side sticky tape, ventral side up. Small pieces of GFP-positive larval brains were transplanted with a constructed glass capillary needle (needle puller- Narishige Japan model PN-30; needles made from Pasteur pipettes of length $230 \mathrm{~mm}$ - Fortuna Cat no. 3.326) tangentially into the mid-ventral abdomen of female host flies. Post recovery from anesthesia, the host flies were maintained at standard conditions at $29^{\circ} \mathrm{C} / 25^{\circ} \mathrm{C}$ or $18^{\circ} \mathrm{C}$ depending on experimental needs.

Surviving flies were transferred to fresh food bottles every second day. Both the surviving and dead host flies were observed under a fluorescent scope once or twice a week (more frequent if required) to assay the formation of tumors. Pictures of transplanted host flies (with or without) tumors were taken with a Nikon Coolpix 4500 digital camera. 
Citation: Laurenson AS, Saini N, Jiang Y, Reichert H (2012) Targeted Transgenic RNAi Knockdown of Cell Fate Determinants Induces Neoplastic Tumor Growth and Metastasis in a Drosophila Transplantation Model of Neural Stem Cell Derived Cancer. J Stem Cell Res Ther S12:002. doi:10.4172/2157-7633.S12-002

Page 3 of 8

\section{Dissection of ovarioles of transplanted flies and Detection of micrometastases}

After tumor formation, the adult abdomens were dissected in Grace's insect medium (1 X, GIBCO, Invitrogen, 11605) at room temperature (RT) and the dissected ovaries were immediately fixed in $4 \%$ paraformaldehyde, $0.2 \%$ of Triton-X-100 dissolved in Grace's insect medium for $30 \mathrm{~min}$ without shaking. The fixative was then rinsed three times in Phosphate Buffer Saline (PBS) $+0.5 \%$ Triton-X-100 (PBST), then washed three times for 10 min each in PBST. Samples were then incubated for $1 \mathrm{~h}$ at RT with Phalloidin-alexa 568 (Molecular Probes, Invitrogen detection technologies) diluted 1:200 in PBST, followed by three washes with PBST for 10 minutes each and a second incubation with Toto-3 iodide (Molecular Probes, Invitrogen detection technologies) diluted 1:1000 in PBST for $1 \mathrm{~h}$ at RT. Samples were rinsed three times in PBST, and washed three times for 10 min each in PBST, rinsed two times in PBS, washed two times for $10 \mathrm{~min}$ each in PBS and then embedded in Vectashield overnight at $-20^{\circ} \mathrm{C}$. Then ovaries were dissected and the separated ovarioles were mounted onto a slide in Vectashield mounting medium. The presence of metastases within ovarioles was detected using a Leica TCS SP5 confocal microscope and the images were processed using standard Fiji [31] or ImageJ 1.42a (NIH, USA).

\section{Results}

Targeted transgenic knockdown of numb, pros and brat in neuroblasts causes overproliferation phenotypes in the larval central brain

Previous work has shown that mutational loss-of-function of the cell fate determinant-encoding genes numb, brat and pros in neuroblasts results in overproliferation in the developing central brain of Drosophila [19-22]. More recent work using insc-Gal4 to drive the expression of transgenic RNAi in central brain neuroblasts indicates that knockdown of numb, brat or pros can replicate the corresponding mutant phenotypes [33]. To confirm this and to document the resulting knockdown phenotypes, we first carried out the corresponding targeted knockdowns for each of these three cell fate determinants in all central brain neuroblasts by using insc-Gal4 to drive the corresponding UASknockdown transgene as well as the UAS-CD8::GFP label to visualize the affected cells and UAS-Dicer2 to enhance the RNAi effect.

In the wild type control (without the UAS-knockdown transgene), all of the neuroblasts and a subset of their progeny are labeled as distinct and well defined cell clusters in the central brain and ventral nerve cord of the third instar larval stage (Figure 1A). (Some labeling is also seen in the developing optic lobes, however, this is not considered further in this report). In the brat knockdown, a dramatic overproliferation occurs in the central brain hemispheres, which is increased significantly in size at the third instar larval stage, and owing to this overproliferation, intense GFP labeling is seen throughout the brain hemispheres (Figure 1B). (This intense fluorescence resulting from the strong GFP labeling masks the normal label of unaffected neuroblasts in the ventral nerve cord). In the numb knockdown situation, a comparable overproliferation is seen in the central brain hemispheres, which are also increased in size at the third instar larval stage and are intensely labeled by GFP (Figure 1C). In the pros knockdown, significant overproliferation is observed in the central brain hemispheres, however, the extent of this overproliferation is markedly reduced as compared to the numb and brat knockdowns in the third instar larval stage (Figure 1D)

Targeted transgenic knockdown can be limited to the type II neuroblast lineages in the central brain by using the worniu-Gal4 driver combined with an asense-Gal80 repressor [33]. To investigate the phenotypes caused by targeted knockdown of numb, brat or pros exclusively in type II central brain neuroblast lineages, we used worGal4, ase-Gal80 to drive the corresponding UAS-knockdown transgene (with UAS-CD8::GFP and UAS-Dicer2). In the wild type control (without the UAS-knockdown transgene), the 8 type II neuroblasts and a subset of their progeny are labeled in each brain hemisphere of the third instar larval stage as distinct cell clusters with corresponding axon tracts (Figure 1E). (Specific labeling is also seen in the developing optic lobes; this is not considered further). In the brat knockdown, a marked overproliferation occurs in the central brain hemispheres of the third instar larval brain (Figure 1F). This overproliferation is characterized by an increased number of labeled cell clusters which contain both large, neuroblast-like cells and numerous smaller cells

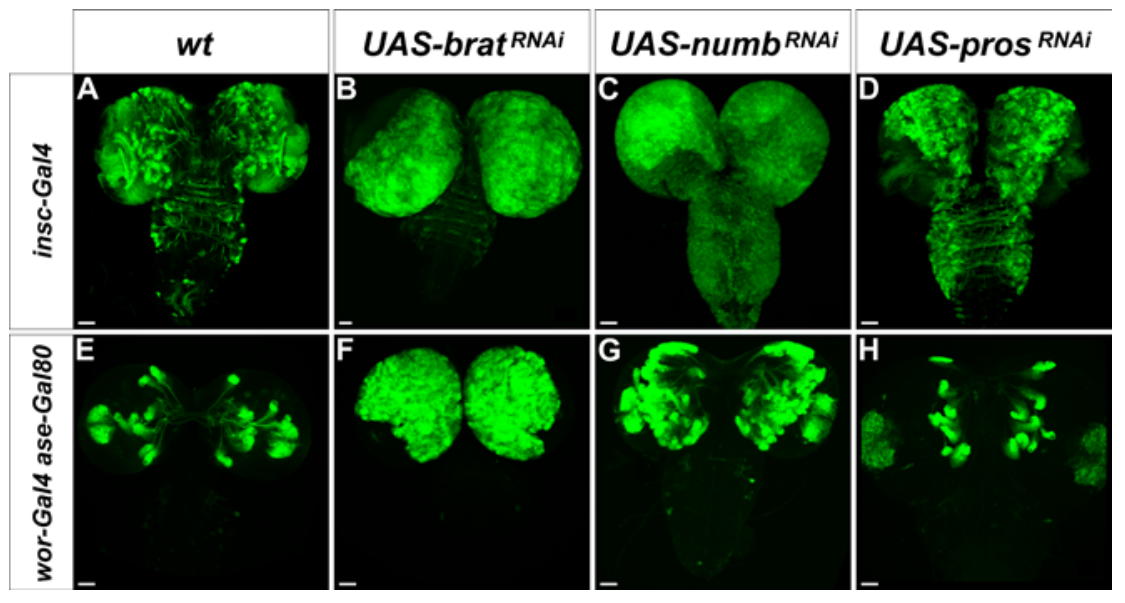

Figure 1: Targeted knockdown of brat, numb, or pros in neuroblasts causes overproliferation in the larval brain. Confocal images of larval brains at late third instar stage labeled with mCD8::GFP (green) of wild type ( $\mathrm{A}$ and $\mathrm{E}$ ), brat knockdown (B and F), numb knockdown (C and G), and pros knockdown (D and H). Targeted knockdown was carried out by using insc-Gal4 driver (A-D) in all neuroblasts or by wor-Gal4 ase-Gal80 driver in only the type II neuroblast lineages (E-H). Scale bars are $50 \mu \mathrm{m}$ 
Citation: Laurenson AS, Saini N, Jiang Y, Reichert H (2012) Targeted Transgenic RNAi Knockdown of Cell Fate Determinants Induces Neoplastic Tumor Growth and Metastasis in a Drosophila Transplantation Model of Neural Stem Cell Derived Cancer. J Stem Cell Res Ther S12:002. doi:10.4172/2157-7633.S12-002

which may correspond to dysregulated INPs. Similarly, in the numb knockdown a significant overproliferation is seen in the central brain hemispheres of the third instar larval brain, and this overproliferation is also characterized by an increased number of labeled cell clusters which contain large, neuroblast-like cells and numerous smaller cells (Figure $1 \mathrm{G})$. In contrast, the pros knockdown shows no overproliferation in the central brain hemispheres; the 8 type II neuroblasts and a subset of their progeny are labeled in each brain hemisphere of the third instar larval stage as distinct cell clusters which correspond to those observed in the wild type control (Figure $1 \mathrm{H}$ ). This result is expected for the pros knockdown as, in type II neuroblast lineages, unlike in type I neuroblasts, the cell fate marker, Prospero is not expressed until the mature INPs stage [34].

These results confirm earlier work and indicate that targeted knockdown of numb, brat or pros in all brain neuroblasts and targeted knockdown of numb or brat in type II neuroblast lineages result in overproliferation phenotypes. Moreover, at least for the knockdown of numb, brat or pros in all brain neuroblasts, the resulting knockdown phenotypes in the late larval brain are comparable to those achieved by the corresponding loss-of-function mutations $[19,23]$.

Tumor formation after transplantation of brain tissue containing RNAi knockdown induced overproliferating brain neuroblasts

The transplantation of brain tissue that is mutant for numb, pros or brat into the abdomen of wild type hosts can result in the formation of tumors that have the potential to grow enormously in size and eventually kill the host [23]. However, it is unknown if brain tissue that overproliferates due to RNAi-induced targeted knockdown of numb, pros or brat in brain neuroblasts can also induce tumors after transplantation. To investigate this, we targeted the RNAi knockdowns to all neuroblasts using insc-Gal4 as well as restricted to type II neuroblast lineages using wor-Gal4, ase-Gal80 and then transplanted the (GFP-labeled) central brain tissue from the corresponding third instar larval stages into the abdomen of adult wild type hosts and assayed for tumor formation. The results of these transplantation experiments are shown in Figure 2.

In the wild type control (without the UAS-knockdown transgene), transplanted brain tissue never resulted in tumor formation when assayed up to five weeks after transplantation. This confirms the fact that the transplantation procedure of wild type GFP-labeled tissue itself is not responsible for the tumorigenesis. In contrast to wild type transplantations and in accordance with reports from previous studies, when brat mutant (loss-of-function) brain tissue was transplanted, tumors resulted in majority of the host flies. In experiments involving targeted insc-Gal4 driven knockdown of numb, brat or pros, transplanted brain tissue also resulted in tumor formation with high frequency. In all three cases GFP-labeled tumors that filled the abdomen of the host were observed in majority of the flies within one week after transplantation, and after two weeks most of these host flies had died. In experiments involving targeted wor-Gal4, ase-Galso driven knockdown of numb or brat, transplanted brain tissue also resulted in tumor formation. However, there was a marked delay in the time needed for tumors to become visible; tumors were only seen in brat-knockdown transplantation experiments after 2 weeks and in $n u m b$-knockdown experiments after 3 weeks. Thus, knockdown of $n u m b$ or brat specifically in type II neuroblast lineages not only results in a more restricted overproliferation of brain tissue, but also results in tumor formation after transplantation that is more delayed in time and/or reduced in frequency of occurrence. (Transplantation of brain tissue from wor-Gal4, ase-Gal80 targeted knockdown of pros did not result in tumor formation; data not shown).

Taken together, these findings demonstrate that targeted knockdown of numb, brat or pros in all central brain neuroblasts results
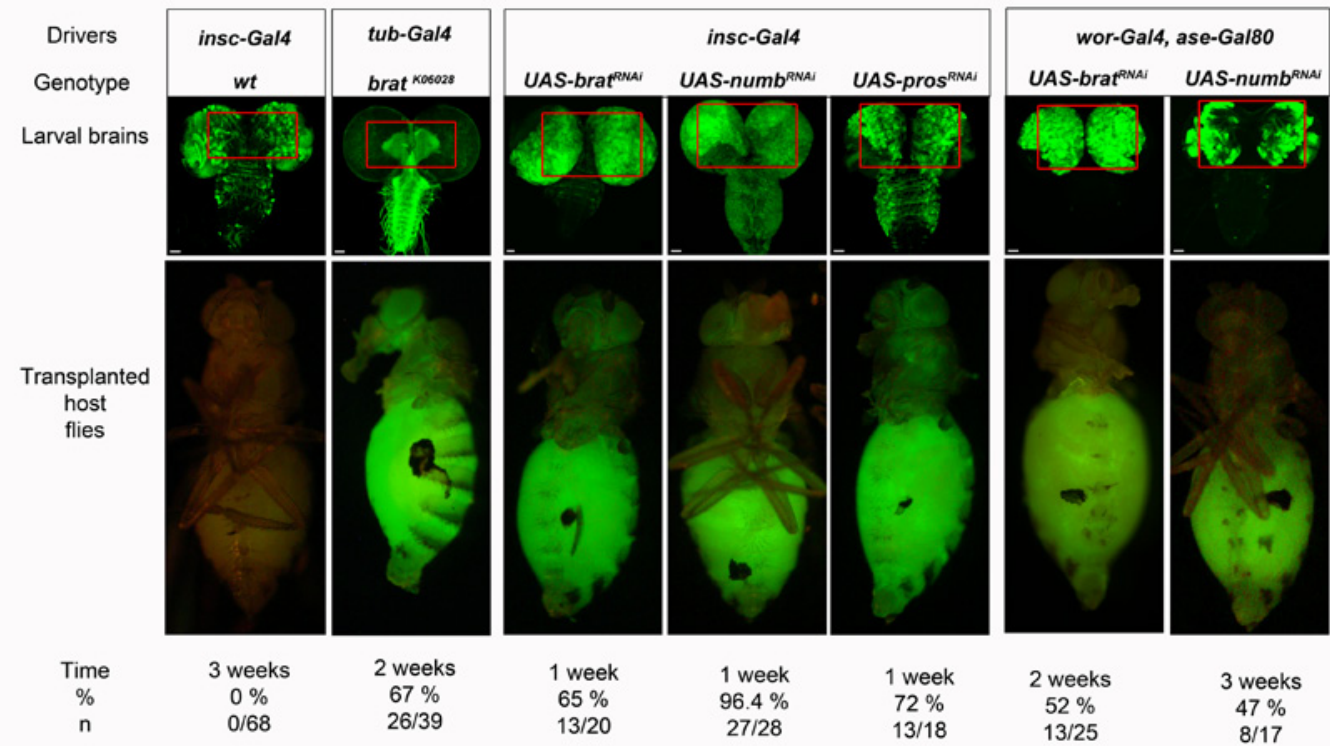

Figure 2: Tumor formation in the abdomen of $w^{1118}$ host flies, after transplantation of overproliferating brain tissue of brat, numb or pros RNAi knockdown. Top panel: confocal images of third instar larval brains labeled with mCD8::GFP (green) with respective genotypes and drivers (scale bars are 50 $\mu \mathrm{m})$. tub-Gal4 (tubulin) drives UAS mCD8::GFP (green) in brat ${ }^{k 06028}$ mutant. Bottom panel: $w^{1118}$ host flies transplanted with pieces of central brain (shown by red outline) of the corresponding genotypes. (Black spot on the abdomen is the scar of the point of injection). The transplantation of bratk06028 mutant; brat, numb or pros RNAi knockdowns, induce tumor formation (green abdomen) in contrast to wild type (wt) control in host flies. ' $n$ ' represents the number of tumor-positive host flies out of the total number of transplanted host flies and the percentage of tumor formation is shown at the respective time point. 
Citation: Laurenson AS, Saini N, Jiang Y, Reichert H (2012) Targeted Transgenic RNAi Knockdown of Cell Fate Determinants Induces Neoplastic Tumor Growth and Metastasis in a Drosophila Transplantation Model of Neural Stem Cell Derived Cancer. J Stem Cell Res Ther S12:002. doi:10.4172/2157-7633.S12-002

Page 5 of 8

in overproliferating brain tissue and that transplantation of this brain tissue can cause a high frequency of tumor formation in host flies which is comparable to that obtained with loss-of-function mutant brain tissue. In view of these findings, we conclude that a targeted transgenic RNAi approach is well suited for investigations of neuroblast-derived tumor formation in the Drosophila transplantation model.

Temperature dependence of tumor formation after transplantation of overproliferating neuroblast tissue in transgenic RNAi-knockdown experiments

The targeted transgenic knockdown of numb, brat or pros in brain neuroblasts is mediated by the Gal4/UAS binary expression system, and this system manifests a small but significant variation of expression as a function of temperature [29]. Moreover, the rate of the cellular overproliferation that characterizes tumors in Drosophila is likely to be temperature dependent as well, although this has not been investigated in a quantitative manner before. Both considerations suggest that the rate and/or frequency of tumor formation after transplantation of overproliferating brain tissue in experiments involving targeted Gal4 driven knockdown of numb, brat or pros, might be temperature dependent. To investigate this in more detail, we determined the frequency of tumors formed in transplantation experiments at $18^{\circ} \mathrm{C}$, $25^{\circ} \mathrm{C}$ and $29^{\circ} \mathrm{C}$ for insc-Gal4 driven knockdown of numb, pros and brat.

Figure 3 shows the temperature dependence of tumor formation after transplantation of brain tissue in which insc-Gal4 driven knockdown of numb was targeted to all neuroblasts. Transplantation of overproliferating brain tissue from third instar larval stages into the abdomen of adult hosts maintained at $29^{\circ} \mathrm{C}$ resulted in large (GFPlabeled) tumors in 27 out of 28 flies (96.4\%) after one week. In contrast, when overproliferating brain tissue was transplanted into adult hosts kept at $25^{\circ} \mathrm{C}$, only 1 out of $26(3.8 \%)$ flies had visible tumors after one week. This rate of tumor formation increased to $50 \%$ of the flies after 2 weeks and $70 \%$ of the flies after 3 weeks. When overproliferating brain tissue was transplanted into host flies kept at $18^{\circ} \mathrm{C}$, no tumors were visible after one or two weeks, and after 3 weeks tumors were visible in only 1 out of 27 (3.4\%) flies. This low rate of tumor formation increased slightly to $7.7 \%$ at four weeks and a substantial number of tumors (41.5\%) were only visible after five weeks at this lower temperature. These results indicate that the frequency of tumor formation is markedly temperature dependent for numb knockdown brain tissue transplants. At $29^{\circ} \mathrm{C}$ virtually all hosts develop visible tumors after one week; at $18^{\circ} \mathrm{C}$ less than $10 \%$ of the hosts develop visible tumors after four weeks.

The corresponding temperature dependence of tumor formation after transplantation of brat or pros knockdown tissue (insc-Gal4 driver) is summarized in Table 1 (pros knockdown) and Table 2 (brat knockdown). In both cases the percentage of visible tumors formed at a given time point after transplantation was highest at $29^{\circ} \mathrm{C}$, intermediate at $25^{\circ} \mathrm{C}$ and lowest at $18^{\circ} \mathrm{C}$. For example, after two weeks in the case of pros knockdown, $80 \%$ of flies had visible tumors at $29^{\circ} \mathrm{C}, 70 \%$ at $25^{\circ} \mathrm{C}$, and $8 \%$ at $18^{\circ} \mathrm{C}$. Similarly, after two weeks in the case of brat knockdown, $83 \%$ of flies had visible tumors at $29^{\circ} \mathrm{C}, 60 \%$ at $25^{\circ} \mathrm{C}$, and $16 \%$ at $18^{\circ} \mathrm{C}$.

These experiments demonstrate that the rate of visible tumor formation after transplantation has a marked temperature dependence in experiments involving targeted Gal4 driven knockdown of numb,

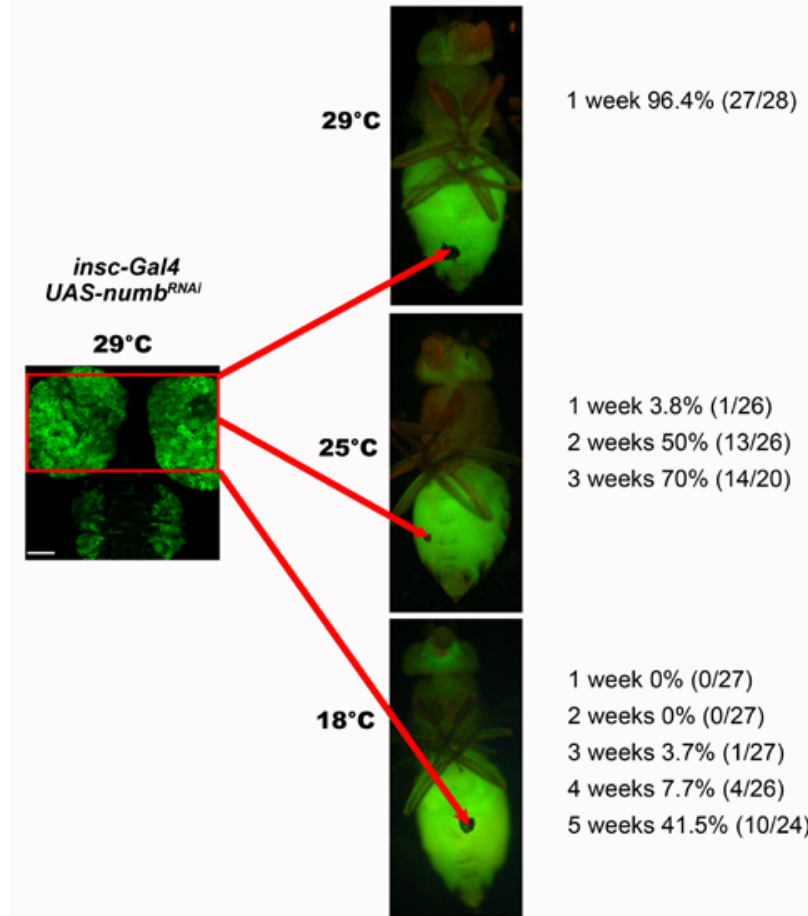

Figure 3: Temperature dependence of tumor formation after transplantation of overproliferating brain tissue of numb-RNAi knockdown. The frequency of tumor formation at different time points (after transplantation) is recorded and displays a marked temperature dependence effect, with maximum frequency of tumor formation seen at $29^{\circ} \mathrm{C}$, intermediate at $25^{\circ} \mathrm{C}$ and minimum at $18^{\circ} \mathrm{C}$.

\begin{tabular}{|c|c|c|c|}
\hline \multicolumn{4}{|c|}{$\begin{array}{c}\text { Temperature dependence of tumor formation in } w^{1118} \text { host flies after } \\
\text { transplantation of third instar larval brain pieces of pros-RNAi knockdown } \\
\text { using insc-Gal4 driver }\end{array}$} \\
\hline Temperature & $\begin{array}{c}\text { Time after } \\
\text { Transplantation }\end{array}$ & $\begin{array}{l}\text { Percentage of } \\
\text { Tumor }\end{array}$ & $\begin{array}{l}\text { Number of tumor- } \\
\text { positive flies/total } \\
\text { number of transplanted } \\
\text { flies }\end{array}$ \\
\hline \multirow[t]{2}{*}{$29^{\circ} \mathrm{C}$} & 1 week & $72 \%$ & $13 / 18$ \\
\hline & 2 weeks & $80 \%$ & $12 / 15$ \\
\hline \multirow{2}{*}{$25^{\circ} \mathrm{C}$} & 1 week & $67 \%$ & $8 / 12$ \\
\hline & 2 weeks & $70 \%$ & $7 / 10$ \\
\hline \multirow{3}{*}{$18^{\circ} \mathrm{C}$} & 2 weeks & $8 \%$ & $1 / 12$ \\
\hline & 3 weeks & $16 \%$ & $2 / 12$ \\
\hline & 4 weeks & $72 \%$ & $5 / 7$ \\
\hline
\end{tabular}

Table 1: pros-RNAi driven by insc-Gal4 in all brain neuroblasts results in an overproliferation, which results in tumor formation in host flies upon transplantation. The frequency of tumor formation at different time points (after transplantation) is recorded and displays a marked temperature dependence effect, with maximum frequency of tumor formation seen at $29^{\circ} \mathrm{C}$, intermediate at $25^{\circ} \mathrm{C}$ and minimum at $18^{\circ} \mathrm{C}$

brat or pros in brain neuroblasts. Highest levels of tumor formation are achieved in shortest time period at $29^{\circ} \mathrm{C}$ indicating that this temperature is well suited for induction of tumors after transplantation in host flies. Nevertheless, even at $18^{\circ} \mathrm{C}$ a relatively high percentage of visible tumors are formed after 4-5 weeks in the numb, pros and brat knockdown transplant experiments. This is of interest for future experiments on mechanisms of tumor formation since $18^{\circ} \mathrm{C}$ is generally the permissive temperature for the use of temperature sensitive genetic control elements such as Gal80 ${ }^{t s}$. 
Citation: Laurenson AS, Saini N, Jiang Y, Reichert H (2012) Targeted Transgenic RNAi Knockdown of Cell Fate Determinants Induces Neoplastic Tumor Growth and Metastasis in a Drosophila Transplantation Model of Neural Stem Cell Derived Cancer. J Stem Cell Res Ther S12:002. doi:10.4172/2157-7633.S12-002

Page 6 of 8

\begin{tabular}{|c|c|c|c|}
\hline \multicolumn{4}{|c|}{$\begin{array}{l}\text { Temperature dependence of tumor formation in } w^{1118} \text { host flies after } \\
\text { transplantation of third instar larval brain pieces of brat-RNAi knockdown } \\
\text { using insc-Gal4 driver }\end{array}$} \\
\hline Temperature & $\begin{array}{c}\text { Time after } \\
\text { Transplantation }\end{array}$ & $\begin{array}{l}\text { Percentage of } \\
\text { Tumor }\end{array}$ & $\begin{array}{l}\text { Number of tumor- } \\
\text { positive flies/ } \\
\text { total number of } \\
\text { transplanted flies }\end{array}$ \\
\hline \multirow[t]{2}{*}{$29^{\circ} \mathrm{C}$} & 1 week & $65 \%$ & $13 / 20$ \\
\hline & 2 weeks & $83 \%$ & $10 / 12$ \\
\hline \multirow[t]{2}{*}{$25^{\circ} \mathrm{C}$} & 1 week & $42 \%$ & $5 / 12$ \\
\hline & 2 weeks & $60 \%$ & $6 / 10$ \\
\hline \multirow{4}{*}{$18^{\circ} \mathrm{C}$} & 2 weeks & $16 \%$ & $2 / 12$ \\
\hline & 3 weeks & $16 \%$ & $2 / 12$ \\
\hline & 4 weeks & $40 \%$ & $4 / 10$ \\
\hline & 5 weeks & $78 \%$ & $7 / 9$ \\
\hline
\end{tabular}

Table 2: brat-RNAi driven by insc-Gal4 in all brain neuroblasts results in an overproliferation, which results in tumor formation in host flies upon transplantation. The frequency of tumor formation at different time points (after transplantation) is recorded and displays a marked temperature dependence effect, with maximum frequency of tumor formation seen at $29^{\circ} \mathrm{C}$, intermediate at $25^{\circ} \mathrm{C}$ and minimum at $18^{\circ} \mathrm{C}$

Tumor invasion and metastasis after transplantation of overproliferating neuroblast tissue in transgenic RNAiknockdown experiments

In the Drosophila tumor transplantation model it is possible to distinguish between non-invasive benign or hyperplastic overgrowth and malignant neoplastic overgrowth which results in invasion and metatstases [25]. Previous work has established an in vivo assay system for metastasis formation by tumor cells which is based on analyzing micrometastases within the ovarioles of adult hosts after transplantation [36]. Since the ovary is surrounded by an epithelial sheet and muscle layers surround the ovarioles, micrometastases can appear in this assay system only if tumorigenic cells leave the transplanted neoplastic tumor mass and actively invade theses cell layers and colonize the host ovarioles [36]. To determine if the tumors caused by transplantation of brain tissue that overproliferates due to RNAi-induced targeted knockdown of numb, pros or brat can lead to micrometastases, we analysed the ovarioles of the transplanted host flies.

Transplantation of wild type control brain tissue (without the UASknockdown transgene) into host flies did not result in visible tumors; ovarioles isolated from these host flies never showed GFP-labeled cells indicating that invasion and metastasis formation did not occur (Figure 4A). In contrast, and as described above, transplantation of brain tissue in which the insc-Gal4 driver was used to knockdown numb, brat or pros in all neuroblasts resulted in visible tumor formation at high frequency in the host. Ovaries isolated from these host flies regularly showed clear micrometastases formation manifest as groups of GFPlabeled cells which invaded and colonized the ovarioles (Figure 4B). Similar findings were obtained for transplantation of brain tissue in which wor-Gal4, ase-Gal80 driver was used to knockdown numb or brat in type II neuroblast lineages; large visible tumors formed and micrometastases developed in the ovarioles of the host flies (Figure 4C). The rate of micrometastasis formation, like the rate of tumor formation, was markedly temperature dependent. The percentage of ovarioles that contained GFP-labeled micrometastases at a given time point after transplantation was highest at $29^{\circ} \mathrm{C}$, intermediate at $25^{\circ} \mathrm{C}$ and lowest at $18^{\circ} \mathrm{C}$ (data not shown).

Taken together, these experiments indicate that the tumors, which are formed after transplantation of overproliferating neuroblast tissue in targeted transgenic knockdown experiments, are neoplastic, invasive and give rise to metastases in the host. This, in turn, implies that the powerful transgenic RNAi approach can be applied to the study of the uncontrolled immortalized proliferation, tumor invasion and metastatic potential of tumors that derive from transplanted dysregulated neuroblasts in the Drosophila model.

\section{Discussion}

For several decades, the Drosophila model system has been successfully applied to the investigation of the molecular basis of cancer, notably due to the ability to carry out large scale genetic screens [13] These screens have led to identification of key regulatory genes which, when mutated, contribute to tumor-like overproliferation in the intact organism and to transplantable tumors with invasive and metastatic properties in host flies $[12,23,25]$. A remarkable finding obtained in the last decade is that mutational inactivation of key regulators of asymmetric cell division of the neural stem cell-like neuroblasts of Drosophila is causal in brain tumor formation implying that defects in neural stem cell asymmetric division can result in tumorigenesis [10,33]. For brain neuroblasts, this has been particularly well documented for mutations in the cell fate determinants numb, brat or pros both in vivo and in the transplantation model of neoplastic tumor formation and metastasis formation [19-22,36]. Thus, at least for these

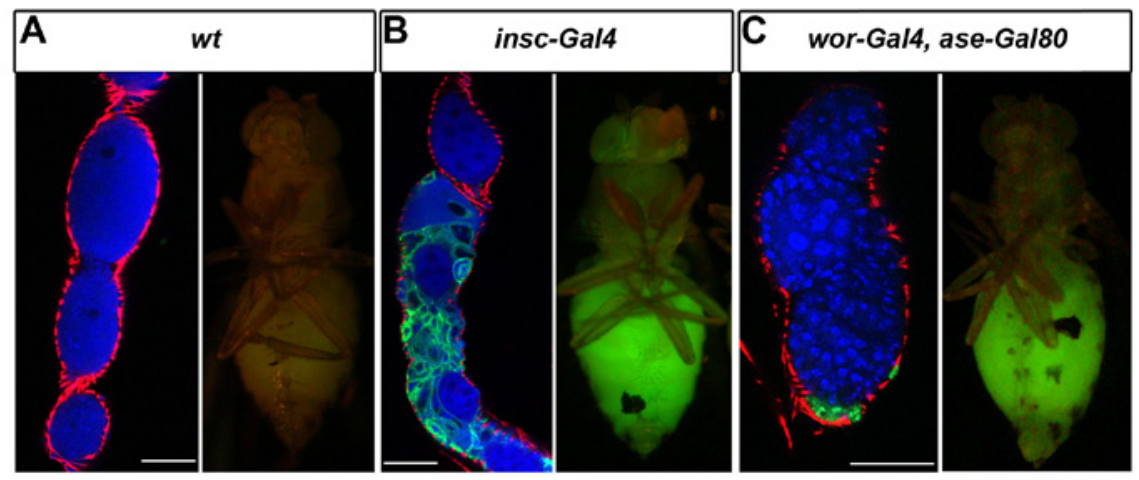

Figure 4: Micrometastasis formation in the ovarioles of host flies, after transplantation of overproliferating brain tissue of numb RNAi knockdown. Confocal images of ovarioles of corresponding $w^{1118}$ transplanted host flies: (A) insc-Gal4 driver control, (B) insc-Gal4 driven numb RNAi and (C) wor-Gal4, ase-Gal80 driven numb RNAi. In (B and $C$ ) the presence of GFP-labeled cells (in green) in the ovarioles indicates the presence of metastasis. DNA is labeled with Toto-3 iodide (in blue) and muscle layers by phalloidin (in red). Scale bars are $30 \mu \mathrm{m}$. 
Citation: Laurenson AS, Saini N, Jiang Y, Reichert H (2012) Targeted Transgenic RNAi Knockdown of Cell Fate Determinants Induces Neoplastic Tumor Growth and Metastasis in a Drosophila Transplantation Model of Neural Stem Cell Derived Cancer. J Stem Cell Res Ther S12:002. doi:10.4172/2157-7633.S12-002

Page 7 of 8

three genes, a solid molecular link between dysregulated neural stem cell proliferation and tumorigenesis has been established in Drosophila.

During the last decade, the arsenal of genetic tools available for investigating the Drosophila model has increased considerably and sophisticated transgenic methods for visualization and manipulation of gene expression have been developed, many of which are based on binary expression systems such as the Gal4/UAS system [38]. Importantly, genome-wide transgenic RNAi libraries for conditional gene inactivation of fly genes, and that can be targeted by the Gal4 system have become available and have been used successfully to identify near-complete sets of genes involved in key cellular processes in vivo [26,27]. Recently, a genome-wide analysis based on transgenic RNAi has been applied to the analysis of the molecular mechanisms of neural stem cell proliferation in Drosophila [30]. In this study, over 600 genes were identified that operate in fly neuroblast proliferation, and the network of key members of this gene population was established, thus documenting the power of a genome-wide analysis of normal and genetically dysregulated neural stem cell proliferation in the intact developing brain.

Here we have applied the same targeted transgenic RNAi approach used by Neumüller and colleagues [30] to the transplantation model of neuroblast-derived neoplastic tumorigenesis and metastasis in Drosophila. Our experiments with targeted knockdown of numb, brat or pros in brain neuroblasts demonstrate for the first time that transgenic RNAi gives rise to phenotypically similar phenomena of neoplastic tumor formation, tissue invasion and metastasis after transplantation as the loss-of-function null mutations of these genes. This implies that a manipulative RNAi-based knockdown approach should be very well adaptable to the mechanistic molecular analysis of neural stem cell-based tumor and metastasis formation in the fly transplantation model. Due to the targeted nature of the knockdown, our experiments already uncover a difference in the potential for transplantable tumorigenesis if the RNAi knockdown is targeted to all neuroblasts or only to type II neuroblast lineages. This demonstrates the potential of a binary Gal4/UAS-based transgenic knockdown approach for analysing cell- and tissue-specific mechanisms of tumor formation. In addition, our experiments document the prominent temperature dependence effect on the rate of knockdown-induced tumor formation after transplantation that is characteristic of this novel transgenic RNAi approach. This is an important prerequisite for the use of conditional temperature-sensitive binary expression system in this neural stem cell cancer model.

Taken together, our findings provide essential basic information for a subsequent large scale genome-wide analysis of the molecular basis of tumor and metastasis formation in Drosophila. As a first step, this will involve the RNAi-based analysis of the numerous new candidate genes recently identified as players in neuroblast proliferation, in tumor and metastasis formation [30]. Moreover, transgenic RNAi knockdown in the transplantation model will make it possible to perform targeted conditional genetic rescue experiments that are aimed at discovering candidate genes and genetic mechanisms for potential abrogation of neuroblast-derived tumors. This type of approach should also allow the combination of several different RNAi lines to test for interactive effects of new candidate genes. In addition, it will be possible to knockdown specific candidate genes in brain tissue transplanted into different mutant host backgrounds to assay for the mechanisms that underlie differential susceptibility of cancer in host organisms. Once the fundamental molecular elements and mechanisms that underlie neural stem cell-derived cancer have been analysed in Drosophila, these approaches should be useful for directing experiments in the field of tumorigenesis and metastases formation in mammalian models.

\section{Acknowledgements}

We thank Jürgen A. Knoblich, the Vienna Drosophila RNAi Center (VDRC) and the Bloomington Drosophila Stock Center for fly stocks; Emmanuel Caussinus and Susanne Flister for great technical help; and all members of the Reichert lab for discussions and assistance. This work was supported by SNSF-NFP63 "Stem Cells and Regenerative Medicine" grant 406340_128006/1 to Heinrich Reichert.

\section{References}

1. Al-Hajj M, Clarke MF (2004) Self-renewal and solid tumor stem cells. Oncogene 23: $7274-7282$

2. Reya T, Morrison SJ, Clarke MF, Weissman IL (2001) Stem cells, cancer, and cancer stem cells. Nature 414: 105-111.

3. Rosen JM, Jordan CT (2009) The increasing complexity of the cancer stem cell paradigm. Science 324: 1670-1673.

4. Gotz M, Huttner WB (2005) The cell biology of neurogenesis. Nat Rev Mol Cell Biol 6: 777-788.

5. Kriegstein A, Noctor S, Martinez-Cerdeno V (2006) Patterns of neural stem and progenitor cell division may underlie evolutionary cortical expansion. Nat Rev Neurosci 7: 883-890

6. Lui JH, Hansen DV, Kriegstein AR (2011) Development and evolution of the human neocortex. Cell 146: 18-36.

7. Morrison SJ, Kimble J (2006) Asymmetric and symmetric stem-cell divisions in development and cancer. Nature 441: 1068-1074.

8. Gonczy P (2008) Mechanisms of asymmetric cell division: flies and worms pave the way. Nat Rev Mol cell Biol 9: 355-366.

9. Knoblich JA (2008) Mechanisms of asymmetric stem cell division. Cell 132 583-597.

10. Knoblich JA (2010) Asymmetric cell division: recent developments and thei implications for tumour biology. Nat Rev Mol Cell Biol 11: 849-860.

11. Yu F, Kuo CT, Jan YN (2006) Drosophila neuroblast asymmetric cell division: recent advances and implications for stem cell biology. Neuron 51: 13-20.

12. Januschke J, Gonzalez C (2008) Drosophila asymmetric division, polarity and cancer. Oncogene 27: 6994-7002.

13. Miles WO, Dyson NJ, Walker JA (2011) Modeling tumor invasion and metastasis in Drosophila. Disease models \& mechanisms 4: 753-761.

14. Doe CQ (2008) Neural stem cells: balancing self-renewal with differentiation Development 135: 1575-1587.

15. Egger B, Chell JM, Brand AH (2008) Insights into neural stem cell biology from flies. Philos Trans R Soc Lond B Biol Sci 363: 39-56.

16. Zhong W, Chia W (2008) Neurogenesis and asymmetric cell division. Curr Opin Neurobiol 18: 4-11.

17. Knoblich JA, Jan LY, Jan YN (1995) Asymmetric segregation of Numb and Prospero during cell division. Nature 377 : 624-627.

18. Hirata J, Nakagoshi H, Nabeshima Y, Matsuzaki F (1995) Asymmetric segregation of the homeodomain protein Prospero during Drosophila development. Nature 377: 627-630

19. Bello B, Reichert H, Hirth F (2006) The brain tumor gene negatively regulates neural progenitor cell proliferation in the larval central brain of Drosophila. Development 133: 2639-2648.

20. Betschinger J, Mechtler K, Knoblich JA (2006) Asymmetric segregation of the tumor suppressor brat regulates self-renewal in Drosophila neural stem cells. Cell 124: 1241-1253.

21. Lee CY, Wilkinson BD, Siegrist SE, Wharton RP, Doe CQ (2006) Brat is a 
Citation: Laurenson AS, Saini N, Jiang Y, Reichert H (2012) Targeted Transgenic RNAi Knockdown of Cell Fate Determinants Induces Neoplastic Tumor Growth and Metastasis in a Drosophila Transplantation Model of Neural Stem Cell Derived Cancer. J Stem Cell Res Ther S12:002. doi:10.4172/2157-7633.S12-002

Page 8 of 8

Miranda cargo protein that promotes neuronal differentiation and inhibits neuroblast self-renewal. Dev cell 10: 441-449.

22. Wang H, Chia W (2005) Drosophila neural progenitor polarity and asymmetric division. Biol Cell 97: 63-74.

23. Caussinus E, Gonzalez C (2005) Induction of tumor growth by altered stem-cell asymmetric division in Drosophila melanogaster. Nat Genet 37: 1125-1129.

24. Caussinus E, Hirth F (2007) Asymmetric stem cell division in development and cancer. Prog Mol Subcell Biol 45: 205-225.

25. Gonzalez C (2007) Spindle orientation, asymmetric division and tumour suppression in Drosophila stem cells. Nat Rev Genet 8: 462-472.

26. Dietzl G, Chen D, Schnorrer F, Su KC, Barinova Y, et al. (2007) A genome-wide transgenic RNAi library for conditional gene inactivation in Drosophila. Nature 448: 151-156.

27. Mummery-Widmer JL, Yamazaki M, Stoeger T, Novatchkova M, Bhalerao $\mathrm{S}$, et al. (2009) Genome-wide analysis of Notch signalling in Drosophila by transgenic RNAi. Nature 458: 987-992.

28. Neumuller RA, Perrimon N (2011) Where gene discovery turns into systems biology: genome-scale RNAi screens in Drosophila. Wiley Interdiscip Rev Syst Biol Med 3: 471-478.

29. Brand AH, Perrimon N (1993) Targeted gene expression as a means of altering cell fates and generating dominant phenotypes. Development 118: 401-415.
30. Neumuller RA, Richter C, Fischer A, Novatchkova M, Neumüller KG, et al. (2011) Genome-wide analysis of self-renewal in Drosophila neural stem cells by transgenic RNAi. Cell Stem Cell 8: 580-593.

31. Schindelin J (2008) Fiji is Just Image J — Batteries Included. Image J User and Developer Conference.

32. Ashburner M (1989) Drosophila: a Laboratory Handbook, Cold Spring Harbo Laboratory Press, Cold Spring Harbor: 243-244.

33. Neumuller RA, Knoblich JA (2009) Dividing cellular asymmetry: asymmetric cel division and its implications for stem cells and cancer. Genes Dev 23: 2675 2699.

34. Weng M, Lee CY (2011) Keeping neural progenitor cells on a short leash during Drosophila neurogenesis. Curr Opin Neurobiol 21: 36-42.

35. McGuire SE, Roman G, Davis RL (2004) Gene expression systems in Drosophila: a synthesis of time and space. Trends Genet 20: 384-391.

36. Beaucher M, Goodliffe J, Hersperger E, Trunova S, Frydman H, et al. (2007) Drosophila brain tumor metastases express both neuronal and glial cell type markers. Dev Biol 301: 287-297.

37. Robinson DN, Cant K, Cooley L (1994) Morphogenesis of Drosophila ovarian ring canals. Development 120: 2015-2025.

38. Venken KJ, Simpson JH, Bellen HJ (2011) Genetic manipulation of genes and cells in the nervous system of the fruit fly. Neuron 72: 202-230.

This article was originally published in a special issue, Neural Stem Cells handled by Editor(s). Dr. Thomas Lufkin, National University of Singapore, Singapore 